\title{
„Da haste den Salat“
}

\section{Lokale Vernetzung gegen Lebensmittelverschwendung}

\author{
Jan Schröter \\ Phoenix Design \\ Kölner Straße 16 \\ 70376 Stuttgart \\ mail@janschroeter.com
}

Prof. Constanze Langer

Fachhochschule Potsdam

Kiepenheuerallee 5

14469 Potsdam

langer@fh-potsdam.de

\author{
Prof. Steffi Hußlein \\ Hochschule Magdeburg Stendal \\ Breitscheidstr. 2 \\ 39114 Magdeburg \\ Steffi.husslein@hs-magdeburg.de
}

\begin{abstract}
Allein in Deutschland landen jedes Jahr 11 Millionen Tonnen Lebensmittel auf dem Müll, obwohl sie noch zum Verzehr geeignet wären: allein $61 \%$ davon aus privaten Haushalten. Gleichzeitig wächst durch die voranschreitende Urbanisierung der Wunsch nach mehr sozialer Vernetzung. Was können wir gegen diese Entwicklung tun? Wie bringt man Menschen dazu, bewusster mit Nahrungsmitteln umzugehen und welche Rolle können soziale Netzwerke dabei spielen? Im Dialog mit Nutzern entstand mittels Personas und Szenarien „neighborSWAP“: Eine App als lokales Einkaufsnetzwerk. Sie ermöglicht kollaboratives Einkaufen und das Teilen übriggebliebener Mahlzeiten, ungenutzter Lebensmittel oder selbst angebauter Waren über eine Sharing-Funktion. Gleichzeitig inspiriert sie den Nutzer zu einer abwechslungsreichen und bewussten Ernährung
\end{abstract}

\section{Keywords}

Foodsharing, Sharing Economy, App, User Centered

\section{Einleitung}

Naht das Verfallsdatum, müssen noch absolut verzehrbare Produkte aus den Supermarktregalen weichen oder verschwinden im privaten Hausmüll. In Deutschland landen im Jahr pro Person über 80 Kilogramm Lebensmittel auf dem Müll. Unbedachtes 
Einkaufen, der fehlende Überblick über Vorräte und die mangelnde Wertschätzung der Lebensmittel sind einige der Gründe für diese vermeidbare Verschwendung. (VIS Bayern 2014, Bundesministerium für Ernährung, Landwirtschaft und Verbraucherschutz 2012) Gleichzeitig findet durch die voranschreitende Urbanisierung eine Anonymisierung der Gesellschaft statt und die Menschen wünschen sich mehr soziale Vernetzung im städtischen Raum. (Horx 2012)

Wie kann diesem Trend entgegengewirkt werden? Wie kann den Menschen ein bewusster Umgang mit Lebensmitteln und Ressourcen nahegebracht werden? Welche Unterstützung wünschen sich die Menschen überhaupt für den Koch- und Zubereitungsprozess? Und wie kann man die Menschen über den Kochprozess zusammenbringen? Mit diesen und anderen Fragen habe ich mich im Zuge dieser Arbeit beschäftigt.

\section{Prozess}

Um Einkaufs-, Koch- und Essgewohnheiten zu analysieren sowie Anknüpfungspunkte für den Konzeptionsprozess zu finden, wurden zunächst Interviews mit Vertretern unterschiedlicher Personengruppen geführt. Neben Erkenntnissen über den Vor- und Zubereitungsprozess brachten die Interviews und die übrigen Recherche ein zusätzliches Bedürfnis zu Tage: Das Teilen nicht benötigter Lebensmittel zur Vermeidung von unnötigen Abfällen. Im Anschluss an die Interviews wurde der gesamte Prozess des Kochens analysiert und in die folgenden Phasen eingeteilt: Planung, Beschaffung, Transport, Lagerung, Zubereitung und der anschließende Verzehr.
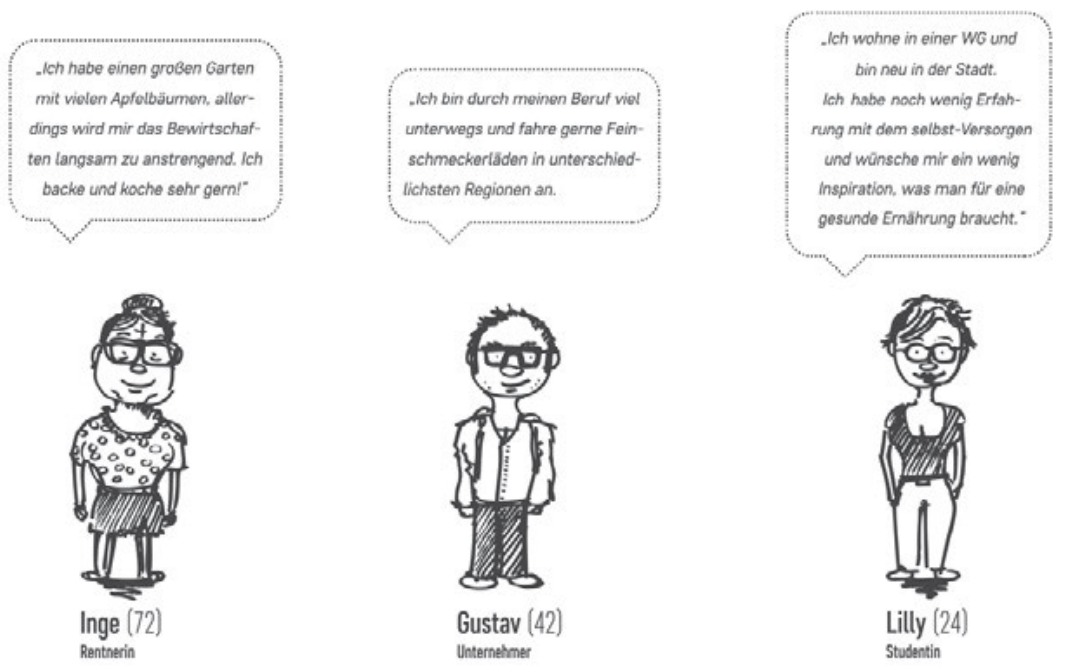

Abbildung 1: Darstellung verschiedener Konsumenten an Hand von Persona 
Um die ermittelten Nutzerbedürfnisse in den Entwurfsprozess einzubringen, wurden Persona entwickelt, die verschiedene Arten von Konsumenten mit verschiedenen Bedürfnissen darstellen. In Abbildung 1 sind drei der fünf Persona dargestellt. Das beispielhafte Durchleben des Organisations- und Kochprozesses an Hand der verschiedenen Persona ergab Aufschluss über wichtige Anforderungen im Prozess der Vernetzung und der Abfallvermeidung in privaten Haushalten.

\section{Ergebnis}

Das Ergebnis der Arbeit ist „neighborSWAP“, eine App, die hilft, übrig gebliebene Speisen, nicht benötigte Nahrungsmittel oder selbst Angebautes in einem lokalen Netzwerk, z.B. unter Nachbarn, zu teilen. Die App ermöglicht zudem das kollaborative Einkaufen innerhalb dieses Netzwerks und fördert saisonale und abwechslungsreiche Ernährung. Damit vereint sie die drei Funktionen „Inspirieren“, „Teilen“ und „Besorgen“. Das Interface ist unterteilt in 3 Bereiche: „Ich brauche“, „Ich teile“ und „Ich besorge“
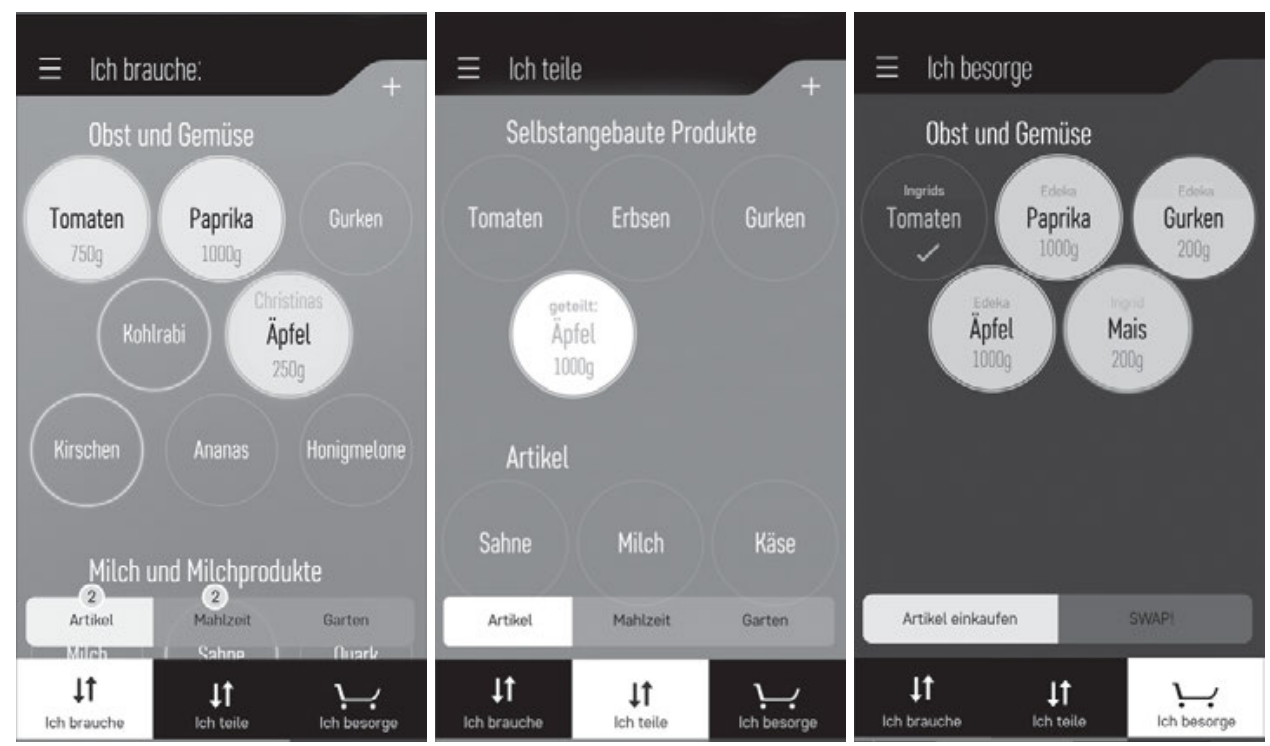

Abbildung 2: Darstellung beispielhafter Screens: Ich brauche, Ich teile, Ich besorge

Ich brauche:

Unter der Kategorie „,ich brauche“ wird eine Liste der regelmäßig gekauften, favorisierten Produkte angezeigt. Die Liste ermöglicht eine schnelle, komfortable Auswahl. Zusätzlich zu den üblicherweise gekauften Produkten werden Empfehlungen wie saisonale Produkte oder in der Nachbarschaft verfügbare Produkte angezeigt. Dies dient dazu, dem Nutzer eine bewusstere, Ressourcen schonendere Ernährung nahezulegen. Hat der Nutzer sehr einseitige 
Produkte in seiner Liste erscheinen zudem abwechslungsreiche, ergänzende Produkte für eine ausgewogene Ernährung in der Liste. Produktempfehlungen sind gekennzeichnet durch eine grüne Umrandung. Waren, die im Netzwerk verfügbar sind, z.B. selbst angebautes Gemüse vom Nachbarn, sind blau umrandet. Eine Rangordnung in der Produktanzeige sorgt dafür, dass Produkte aus dem Netzwerk bevorzugt angezeigt werden.

Ich teile:

Im Bereich ,ich teile“ kann der Nutzer Produkte im Netzwerk für andere sichtbar und verfügbar machen. Dies können selber angebaute Produkte sein oder aber überschüssige Mahlzeiten oder nicht benötigte Lebensmittel.

Ich besorge:

Diese Liste wird beim tatsächlichen Einkauf angezeigt. Es erscheinen die Produkte, die im Bereich „Ich brauche“ ausgewählt wurden, aber auch Produkte, die Nachbarn aus dem Netzwerk gerne aus dem entsprechenden Shop mitgebracht bekommen würden. Über den „SWAP“ Button wird der Austausch von Waren innerhalb des Netzwerkes organisiert.

„neighborSWAP“ soll zu einem bedachteren Konsum von Nahrungsmitteln anregen. Nur wenn sich das Bewusstsein der Menschen im Umgang mit Lebensmitteln ändert, kann etwas gegen die unnötige Verschwendung getan werden. Ich hoffe mit dieser Arbeit einen Anreiz zum Nach- und Umdenken zu geben.

\section{Literatur}

Horx, M. (2012) Megatrend Dokumentation - Statistiken und Prognosen für die Wirtschaft von morgen. zukunftsInstitut (Hrsg.)

Horlemann G. (2014) Lebensmittel wegwerfen, das muss nicht sein! VerbraucherService Bayern. http:/www.vis.bayern.de/ernaehrung/lebensmittel/gruppen/lebensmittelwegwerfen.htm [18.05.2014]

Bundesministerium für Ernährung, Landwirtschaft und Verbraucherschutz (2012) Ermittlung der Mengen weggeworfener Lebensmittel und Hauptursachen für die Entstehung von Lebensmittelabfällen in Deutschland https://www.zugutfuerdietonne.de/uploads/media/Studie_Faktenblatt_01-1_01.pdf [24.06.15] 